\title{
Development of a Selective Extinction through Cognitive Evaluation
}

\section{PARADIGM}

\author{
AUTHORS: \\ Birthe Macdonald ${ }^{1,2}$ \\ Tom Johnstone ${ }^{1,3}$ \\ ${ }^{1}$ University of Reading, Whiteknights, Reading RG6 6AL, UK \\ ${ }^{2}$ University of Zurich, Andreasstrasse 15, 8050 Zurich, birthe.macdonald@uzh.ch \\ ${ }^{3}$ Swinburne University of Technology, John Street, Hawthorn, Victoria 3122, Australia
}

\section{ABSTRACT}

In this study we trialled a novel paradigm that aims to bridge the gap between the research into the extinction of conditioned responses and the instructed regulation of emotion via a prescribed strategy. This was done through a process we called Selective Extinction through Cognitive Evaluation (SECE). Participants were conditioned to associate a previously neutral stimulus (letter, $\mathrm{CS}+$ ) with an aversive burst of white noise (US) and another letter with no aversive event (CS-). In a second phase, the same letters were presented within words belonging to two distinct categories. One group did not receive any more noise bursts during this phase which lead to the extinction of the conditioned responses (extinction group). The other group (SECE group) was told that the CS+ now only carried a risk of the noise occurring when it was presented in one (dangerous), but not the other word category (safe). Affective responses were assessed using skin conductance responses (SCR). We found overall increased SCR's in response to CS+ (safe and dangerous) compared to CS- trials in the SECE group, and also increased SCR's in response to dangerous compared to safe CS+ trials. This suggests that the conditioned response (CR) can be reduced through the cognitive evaluation of additional information and it is possible that extinction mechanisms are involved in this process. We did not find any effects in the extinction group. It is possible that extinction was completed too quickly so that any differences between conditions could not be captured.

We therefore conducted a second study to pilot an improved paradigm. We visually amended the trials, amended the reinforcement schedule, and changed to a fully within-subjects design. Here we once again found significantly reduced SCR to safe compared to dangerous CS+ trials.

Future experiments will use these results as a basis for an improved paradigm to better capture changes in SCR as well as BOLD fMRI in future experiments. 


\section{INTRODUCTION}

Classical conditioning occurs when a previously neutral stimulus (conditioned stimulus, CS+) is paired with an affective stimulus (unconditioned stimulus, US) until the CS+ elicits the same affective response as the US (conditioned response, CR) (Pavlov, 1927). This can be carried out using either positive stimuli such as food, leading to approaching or the strengthening of desired behaviours, or negative stimuli such as a loud noise or an electric shock, leading to conditioned fear and avoidance behaviours such as freezing. The reduction of these conditioned responses is typically achieved through extinction, when the CS+ is repeatedly presented without the US until the CS+ ceases to evoke a CR. The extinction of conditioned aversive responses is an implicit and well-controlled way of testing how affective responses are reduced. In humans, this is often measured through physiological measures such as skin conductance responses (SCR, see Delamater, 2010 for a review).

This extinction effect does not seem to involve the abolition of the CS+-CR relationship, however. Animals that undergo conditioning and then extinction of the $\mathrm{CR}$ one day will tend to show recovery of the CR the following day, albeit to different degrees (see Bouton (2002), for a review). This suggests that a trace of the CS - CR relationship is maintained through extinction, but downregulated by another system responsible for the extinction effect. Thus, extinction is a form of fearrelated learning that involves storing a modified representation of the affective meaning of the previously conditioned CS, and using this to regulate or override the CR.

There are several strands of research that examine variations of the classic extinction paradigm by manipulating the contingencies under which the CS+ is removed. One example which investigates the effects of combining a CS+ and CS- on the CR is 'conditioned inhibition' (Rescorla, 1969) and describes the summation of a CS that reliably predicts a US and another CS which has never been associated with the same US. When presented together, they produce a reduction in CR and a delay in fear conditioning to the previously established CS-. Using SCR and the startle reflex as measures of the CR, Grillon and Ameli (2001) found a reduction in SCR when a previously reinforced CS+ was preceded by a safety signal in low anxious participants. Kong, Monje, Hirsch, \& Pollak (2014) reviewed studies investigating the neural pathways underlying 'Learned Safety', conditioned inhibition specific to aversive US'. This typically involves training participants to associate one stimulus (CS+) with an aversive event and another with the absence, or a period of safety from this aversive event. In a test phase, both are combined and result in a reduced reaction to this combined stimulus. Thus, evidence exists that a previously established CR can be modified and inhibited without an extinction phase, through the addition of safety cues.

It is often assumed that such extinction processes are the basis of higher level cognitive reappraisal of emotion, which serves the same goal as the extinction of conditioned responses: to reduce an 
affective response. However, few studies have been published that explicitly attempt to bridge this gap. Delgado, Nearing, Ledoux, and Phelps (2008) investigated the instructed regulation of conditioned responses to coloured squares. They instructed participants that either a blue or a yellow square indicated a chance that an electric shock would occur. Before each trial participants were instructed to either "attend" to their naturally occurring response to that trial, or to "reappraise" is by thinking of something calming in nature related to the colour. They found that SCR's were higher during CS+ "attend" than CS- "attend" trials, a difference that was abolished for CS+ "reappraise" and CS- "attend" trials. On a neural level, they found increased amygdala activation during CS+ "attend" trials compared to CS+ "reappraise" trials, and vmPFC and dIPFC in the opposite contrast. They concluded that participants were able to regulate their affective response when asked to do so, and that they recruited a set of brain areas that was more specific than that recruited in instructed emotion regulation studies, but extended compared to extinction. This supports the notion that the basic mechanisms involved in the extinction of affective responses and the instructed regulation of emotion overlap. Several questions arise when considering the design of this task. Instead of a standard conditioning procedure, Delgado et al. (2008) used explicit instructions to inform participants of the $\mathrm{CS}+$ /- contingencies. Although it has been shown that affective responses can reliably be evoked in this way, it is unclear whether the two procedures really tap into the same mechanisms. Although it has been shown that affective responses can reliably be evoked in this way, the underlying neural mechanisms are overlapping but not the same (see Mechias, Etkin, \& Kalisch, 2009 for a meta-analysis). In this study, we used a conditioning procedure.

We aimed to develop a version of fear extinction in which a simple cognitive manipulation would enable participants to predict whether a trial including a previously conditioned CS+ is "safe" or "dangerous". Thus, in contrast to the studies mentioned above, it is not an additional CS associated with safety from an aversive event or an explicit instruction which determines whether the CS+ will be followed by the US, but additional information that participants need to cognitively evaluate in order to determine the threat value of the CS+. Because the meaning of the same stimulus that would ordinarily be seen as threatening is changed via a cognitive evaluation of additional information in this study, this strategy could be seen as a simplified version of those used in cognitive reappraisal studies, during which participants are often asked to reappraise aversive images in order to regulate their emotions (McRae, Ciesielski, \& Gross, 2012).In these tasks, participants are asked to employ predefined strategies to change their appraisal of the images they see. Both the images and these strategies are complex and involve a number of cognitive processes to be completed successfully. This new paradigm aims to simplify the process and tap into the basic mechanisms that may be part of complex reappraisal. 
We established a CR in participants through a standard Pavlovian conditioning procedure using letters ( $B$ and $T$ ) as conditioned stimuli. This was followed by a modified extinction phase during which these letters were presented within words belonging to two categories (birds and vegetables). This phase will be titled "Selective Extinction through Cognitive Evaluation (SECE) phase" from here onwards. One of those categories was safe, i.e. no US would be delivered. Thus, the words needed to be cognitively evaluated to determine whether or not the US could be expected to occur subsequently to the CS+ or whether the CS+ could be reinterpreted as safe. The use of letters and words in this task is an attempt to tap into sematic processes during emotion regulation. Instructed emotion regulation tasks often use verbal strategies such as "think of a more positive outcome to the scenario depicted" (McRae et al., 2012), thus, this seemed to be an appropriate starting point for this investigation. However, we designed this task with an emphasis on its simplicity and potential for adaptation, to enable researchers to create different versions of this task to investigate different specific processes that may be involved in emotion regulation, and could be altered in patients with mental disorders who have difficulties regulating their emotion. In this particular method, the cognitive processes involved are limited to word categorisation, remembering safe and dangerous categories, and making a decision about each trial. Other versions of this task might include a different type of safe vs dangerous contingencies, e.g. a more memory based task, or a more abstract visuospatial task.

Affective responses were assessed using skin conductance. Response patterns were then compared to a version of this task which was more similar to classical extinction to determine similarities and differences between the two versions of the task.

To check for possible differences between the two groups of participants that may affect their performance on this task, we also gave participants a number of questionnaires to assess their habitual emotion regulation strategies (Emotion Regulation Questionnaire, ERQ, Gross \& John, 2003), their positive and negative affect (Positive And Negative Affect Scales - now, PANAS-NOW, Watson, Clark, \& Tellegen, 1988) as well as tendency to worry (Penn State Worry Questionnaire, PSWQ, Meyer, Miller, Metzger, \& Borkovec, 1990).

Skin conductance responses (SCR's) are a standard way of assessing autonomic affective responses in humans. In conditioning studies, SCR's tend to rise as the association with the CS+ is established, and fall when the association is taken away (Davey, 1992). We hypothesized that the new paradigm would enable participants to differentiate between safe and dangerous CS+ trials, resulting in a reduction of the CR. Thus, we predict that SCR's will be reduced during safe CS+ trials but maintained high during dangerous CS+ trials. In the group that received a version of the task that was more similar to a classical extinction, we expected to see higher SCR's in response to both categories of CS+ 
compared to CS- trials at the start. We expected this difference to be abolished at the end of the experiment once extinction had occurred.

\section{STUDY 1}

\section{METHOD}

\section{PARTICIPANTS}

42 participants were tested in total, divided into 2 groups of 21 - one group that received the Selective Extinction through Cognitive Evaluation (SECE) part of the task (mean age $=25.4,11$ female), and one that received an extinction phase instead (mean age $=21.6,16$ female). Participants were recruited through the University of Reading Research Panel, flyers around the University of Reading, emails sent to different departments and word of mouth. Participants were randomly assigned to the two groups. All participants were psychologically healthy and had never had a diagnosis of a mental disorder. Participants were reimbursed for their time with a small monetary reward or course credit. This study was approved by the University of Reading Ethics committee (UREC 13/32). All participants provided informed consent and were fully debriefed upon completion of the study.

\section{THE TASKS}

\section{CONDITIONING}

Both groups received the same conditioning procedure during which one of two letters ( $B$ and $T$ ) were paired with a burst of white noise, $100 \%$ of the time. The letter used as CS+ was counterbalanced between participants.

10 CS+ and 10 CS- trials were shown for $2000 \mathrm{~ms}$, with white noise onset $500 \mathrm{~ms}$ into stimulus presentation with a $100 \%$ reinforcement schedule of the CS+.

After this first phase both groups were told that they would now see the same letters within words that belong to two categories (birds and vegetables). They were instructed to monitor the stimuli by pressing one button if the word belonged to one of the two categories and contained the CS+, and the other button if it belonged to one of the categories and contained the CS-. Two filler words which belonged to neither category were included to keep participants alert. This task was added to ensure participants paid attention to both the letter and the word categories. All instructions were delivered through an Eprime script.

\section{Selective Extinction through Cognitive Evaluation}

To induce a reappraisal process, participants in the reappraisal group were told that they would not hear the white noise when the word they viewed belonged to one of the two possible categories. 
The reinforced letter as well as category were counterbalanced between participants.

During the Reappraisal phases, stimuli were presented in a random order and each stimulus was shown for $4000 \mathrm{~ms}$. Stimuli belonging to the "dangerous" category and containing the CS+ were accompanied by white noise after 1500ms. 42 trials were shown, 10 each of dangerous CS+, safe CS+, dangerous CS-, and safe CS- trials (see table 2). Thus, the reappraisal group were shown 10 CS+ within a "safe" word during which no white noise was to be expected. 10 CS+ within a "dangerous" word were also shown, 5 of which were non-reinforced. These were used for analysis.

2 distractor items were also shown to keep participants engaged and ensure they were paying attention to both letter and word stimuli.

The inter trial interval (ITI) was jittered between $2000 \mathrm{~ms}$ and $6000 \mathrm{~ms}$ in preparation for a future fMRI experiment.

\section{EXTINCTION}

Participants in the extinction group were not given any further instructions about the contingencies of the task. They were then presented with the same paradigm as the reappraisal group (i.e. they were also asked to press one button if the word belonged to one of the two categories and contained the CS+, and the other button if it belonged to one of the categories and contained the CS-, as well as withhold their button press for the filler words) but no further noise bursts were delivered.

\section{Apparatus AND MATERIALS}

Stimuli were presented using EPrime 2.0 (Psychology Software Tools, Pittsburgh, PA). White noise was delivered through a set of KYE Systems Genius SP-G06 Hi-Fi Speaker Systemat a level of 90dB.

The two letters used as CS' were " $B$ " and " $T$ ". To provide the safe or dangerous context, 20 different words were chosen from 2 categories: vegetables and birds. The words were chosen on the basis of lists defined as typical members of these categories (Battig \& Montague, 1969). Further words were chosen through online dictionaries. All words were validated by checking their frequency in the English language in a list based on the British National Corpus (Kilgarriff, 1997, for frequencies see table 1).

TABle 1. Means and standard deviations in WORD fRequency baSed on the British National Corpus (KILGARIFF, 1997). NO SIGNIFICANT DIFFERENCE WAS FOUND BETWEEN GROUPS OF WORDS WHEN THEY WERE DIVIDED BY LETTER $(T(18)=1.78, P=0.092)$ OR BY CATEGORY $(T(10)=-1.75, P=0.1)$.

\begin{tabular}{lllll} 
& & Bird & Vegetable & Total \\
\hline & & Mean (SD) & Mean (SD) & Mean (SD) \\
T & Mean (SD) & $211.2(167.09)$ & $374.72(282.33)$ & $292.7(234.98)$ \\
B & Mean (SD) & $79.2(63.44)$ & $208.6(135.27)$ & $143.9(120.72)$ \\
Total & Mean (SD) & $145.2(137.97)$ & $291.4(226.22)$ & \\
\hline
\end{tabular}


Letters and words were presented on a grey background. Stimuli were prepared using PowerPoint. Letters and words were arranged in the centre of each slide, with letters in font size 66 and bold, and words in font size 44 (not bold, see figure 1).

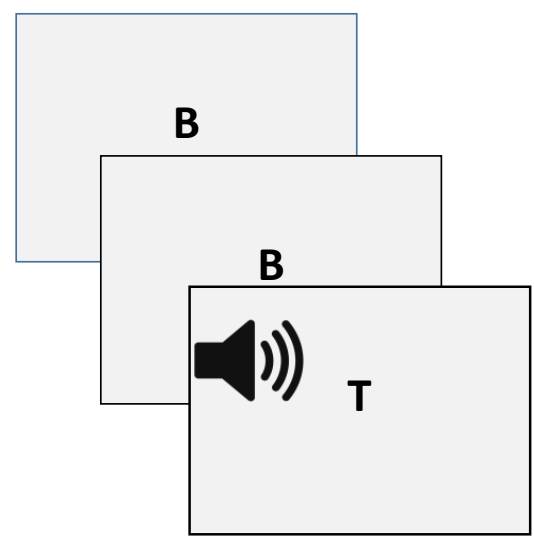

Phase 1 - Conditioning

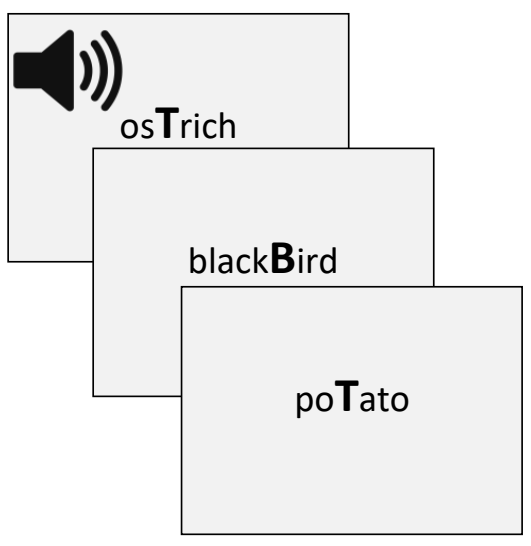

Phase 2 - SECE

FIGURE 1. TRIALS IN THE SECE TASK. LETTERS ARE SHOWN IN BOLD IN THE MIDDLE OF THE SCREEN THROUGHOUT. IN THE SECE PHASE, LETTERS ARE EMPHASIZED WITHIN THE WORDS. IN THE EXTINCTION GROUPS, TRIALS WERE IDENTICAL BUT NO FURTHER SOUNDS WERE DELIVERED.

Skin conductance was recorded with Labchart 7 (ADInstruments, Dunedin, NZ) at a sample rate of $1000 \mathrm{hz}$, using two stainless steel electrodes attached to the distal phalanges of participants' index and middle fingers of their non-dominant hand (Cacioppo, Tassinary, \& Berntson, 2007). A low constant-voltage AC excitation of $22 \mathrm{mVrms}$ at $75 \mathrm{~Hz}$ was passed through the electrodes, which were connected to a ML116 GSR Amp, and converted to DC before being digitized and stored.

A questionnaire with a single question was used to assess the aversiveness of the white noise burst. Participants were required to mark on a 10-point Likert scale whether the sound was 1 (very aversive) to 10 (not aversive at all).

TABLE 2. DESIGN OF THE SECE TASK.

\begin{tabular}{ll|ll|lc}
\hline \multicolumn{2}{c|}{ Conditioning } & SECE Group & & \multicolumn{2}{|l}{ Extinction Group } \\
& & Safe & Dangerous & \multicolumn{2}{l}{ Birds V Vegetables } \\
& (birds or vegetables) & (birds or vegetables) & no further reinforcement \\
CS+ 10 (all reinforced) & 10 & 10 (5 non-reinforced) & 10 & 10 \\
CS- 10 & 10 & 10 & 10 & 10 \\
\hline
\end{tabular}




\section{DESIGN}

The design of this task was different for the two groups. In the reappraisal group we used a univariate design with 3 levels: CS+dangerous vs CS+safe vs CS-. In the extinction group we used a 2 factor design: CS+ vs CS-. Both groups underwent the same conditioning procedures but went on to complete either an extinction or a reappraisal procedure (see table 2.). Types of trials were compared within groups, contrasting (non-reinforced) CS+dangerous, CS+safe and CS- trials in the reappraisal group, and CS+ and CS- trials in the extinction group (all trials were non-reinforced in this group). Because we expected the responses to the CS+ to be reduced slowly in the extinction group, we included a time factor in a second step. Trials were presented in a random order throughout this task. Participants were randomly assigned to either the reappraisal or the extinction groups. CS+ and CS- as well as the safe and dangerous categories were counterbalanced between participants.

\section{PROCEDURE}

After providing informed consent, participants were asked to rate the white noise and complete a number of questionnaires prior to commencing the task assessing their perception of the white noise burst and their participants' emotion regulation strategies, positive and negative affect, tendency to worry, and trait anxiety. The task and data collection procedures were explained and electrodes attached to participants' fingers. To avoid movement artefacts, participants were asked to remain as still as possible throughout. The task was then started and the experimenter left the room. After the task, the experimenter returned and removed the electrodes before debriefing the participant and answering any further questions.

\section{SCR DATA ANALYSIS}

Data was visually checked for motion artefacts and these were removed manually. SCR data was filtered using a band pass filter with a high cutoff of $1 \mathrm{hz}$ and a low cutoff of $0.01 \mathrm{hz}$ using a programme developed by Prof. Tom Johnstone, University of Reading (Johnstone, T. (2017, September 8). Psychophysiology Analysis Software. Retrieved from osf.io/4wsm3). Data was then analysed using a MATLAB (Mathworks Ltd.) script to identify the highest deflection from a 2 second pre-stimulus baseline within a 7 second window after each stimulus onset. Outliers were removed by transforming data into $z$-standardised scores across conditions and removing individual values that exceeded 3.29 or were smaller than -3.29 , i.e. were larger or smaller than $99.99 \%$ of the values obtained in this experiment. Data from 3 participants in the extinction group was removed entirely (1 did not show responsivity throughout the task, 1 due to noise, and 1 due to a technical problem during data collection). Residuals were normally distributed, therefore data was not transformed but original data was analysed. 


\section{RESULTS}

\section{QUESTIONNAIRES}

No group differences were found on any of the three questionnaires (ERQ, PANAS-now, PSWQ) or the stimulus aversiveness ratings (average aversiveness rating $=2.85$ ). Only one participant rated the stimulus higher than 5 (See table 3 for Questionnaire scores).

TABle 3. MeAn Questionnaire sCores.

\begin{tabular}{llllll}
\hline Questionnaire & $\begin{array}{l}\text { SECE } \\
\text { Mean (SD) }\end{array}$ & $\begin{array}{l}\text { Extinction } \\
\text { Group } \\
\text { (SD) }\end{array}$ & $\begin{array}{l}\text { Cronbach's } \\
\text { Alpha }\end{array}$ & T(37) & p \\
Stimulus & $2.67(1.06)$ & $2.83(1.54)$ & & -0.4 & 0.69 \\
Evaluation & & & & & \\
ERQ reappraisal & $29.57(5.02)$ & $28.61(6.36)$ & 0.8 & 0.53 & 0.6 \\
ERQ suppression & $14(4.53)$ & $13.28(4.99)$ & 0.75 & 0.47 & 0.64 \\
PANAS positive & $29.62(6.73)$ & $25.5(7.06)$ & 0.88 & 1.86 & 0.07 \\
PANAS negative & $12.62(3.65)$ & $11.38(1.82)$ & 0.8 & 1.36 & 0.2 \\
PSWQ & $49.05(17.33)$ & $43.78(15.84)$ & 0.93 & 0.98 & 0.3 \\
\hline
\end{tabular}

\section{BehaVIOURAL DATA}

All participants were able to correctly perform the behavioural task throughout at above $80 \%$ accuracy (mean SECE group $=0.85, \mathrm{SD}=0.13$; mean extinction group $=0.87, \mathrm{SD}=0.17$ ). This difference was not significant between the two groups $(t(40)=0.58, p=0.57)$.

\section{CONDITIONING CHECKS}

No reliable comparison could be made between SCR's in response to CS+ and CS- during the conditioning phase, as the CS+ was $100 \%$ reinforced and thus, contaminated by the white noise.

\section{SECE GROUP}

Only trials which did not include a burst of white noise were used in this analysis. A mixed effects GLM with 3 levels (CS+dang vs CS+safe vs CS-) was carried out on the SCR data from the reappraisal group which showed a significant effect of condition $\left(F(2,20)=7.59, p=0.002\right.$, partial $\eta^{2}=0.27$, see figure 4 , and table 4). Because the number of trials in each cell were unequal, sphericity could not be assumed in this case (Mauchly's W $=0.703, p=0.35$ ). Greenhouse-Geisser Correction of the main effect of condition still returned a significant result $\left(F(2,20)=5.81, p=0.012\right.$, partial $\left.\eta^{2}=0.23\right)$. Posthoc tests of this SCR data revealed that this effect was driven by the difference between both levels of CS+ and CS- (Responses to CS+dang was significantly greater than CS-: $F(1,20)=9.92, p=0.005$, Cohen's $d=0.29$; Responses to CS+safe were significantly greater than CS-: $F(1,20)=6.9, p=0.02$, Cohen's $d=0.19$ ). In addition, SCR's to dangerous CS+ trials were significantly larger than those to 
safe CS+ trials $(F(1,20)=3.26, p=0.043$ (one-tailed), Cohen's $d=-0.17$, see figure 2 ).

Participants' questionnaire scores did not have an effect on these results.

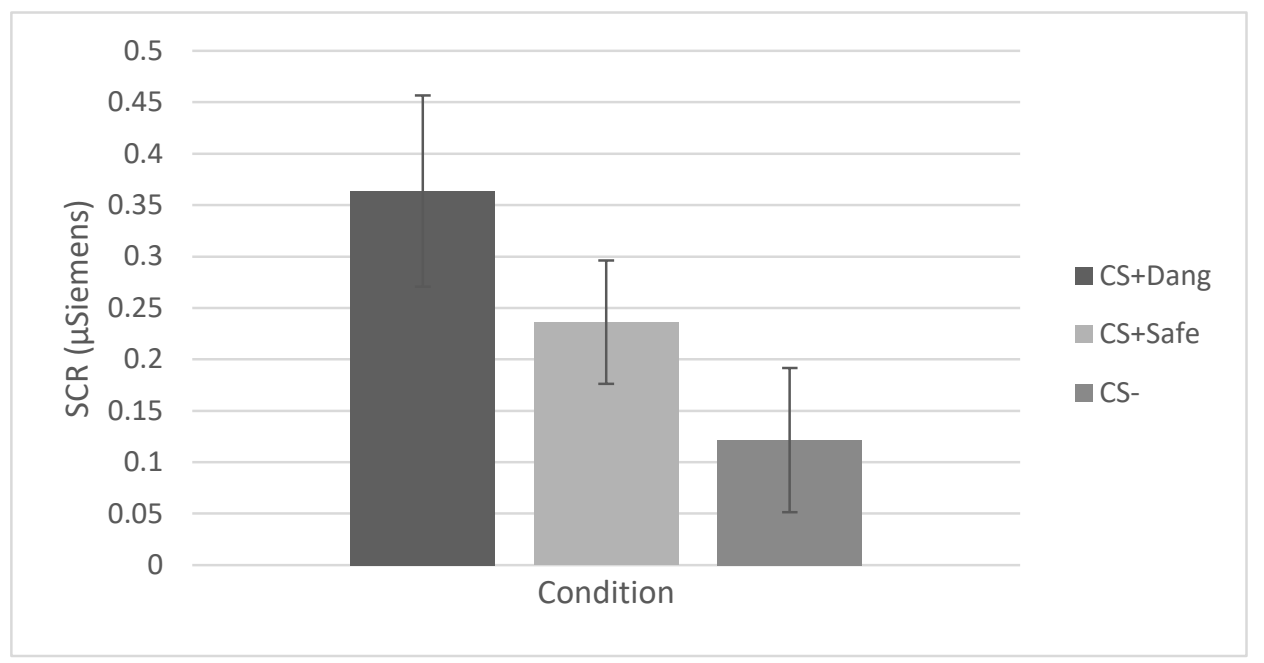

FIGURE 2. SCR IN THE SECE GROUP. ERROR BARS REPRESENT WITHIN SUBJECT 95\% CONFIDENCE INTERVALS.

\section{EXTINCTION GROUP}

A mixed GLM with 2 levels (CS+ vs CS-) was carried out on the data from the extinction group. No significant difference was found between the two conditions $(F(1,17)=0.29, p=0.6$, partial $\left.\eta^{2}=0.013\right)$. The addition of a time factor revealed no significant effect of time $(F(1,17)=0.73, p=0.41$, partial $\left.\eta^{2}=0.001\right)$ and no condition $x$ time interaction $\left(F(1,17)=0.12, p=0.73\right.$, partial $\left.\eta^{2}=0.007\right)$. To check whether there was a difference between CS+ and CS- trials in the first half of the extinction phase, we ran a separate mixed effects GLM on these trials and found no difference $(F(1,17)=0.22, p$ $=0.64$, see table 4 for means).

Participants' questionnaire scores did not have an effect on these results.

TABLE 4. MEAN SCR IN $\mu$ SIEMENS FOR EACH CONDITION. SCR ASSOCIATED WITH DANGEROUS CS+ TRIALS WAS SIGNIFICANTLY INCREASED COMPARED TO RESPONSES TO SAFE CS+ AND CS- SCORES. RESPONSES TO SAFE CS+ TRIALS WERE ALSO SIGNIFICANTLY GREATER THAN RESPONSES TO CS- TRIALS.

\begin{tabular}{lll}
\hline \multirow{2}{*}{ Reappraisal Group } & Condition & Mean $(\mathrm{Cl})$ \\
& & \\
& CS+safe & $0.24(0.16)$ \\
& CS+dang & $0.36(0.16)$ \\
Extinction Group & CS- & $0.12(0.12)$ \\
& & \\
& CS+ & $0.21(0.09)$ \\
& Early & $0.22(0.12)$ \\
& Late & $0.21(0.12)$ \\
& CS- & $0.19(0.09)$ \\
& Early & $0.19(0.12)$ \\
& Late & $0.21(0.12)$ \\
\hline
\end{tabular}




\section{STUDY 1}

\section{DISCUSSION}

The current study aimed to test a novel paradigm to investigate the specific mechanisms involved in emotion regulation. We used two letters (B and T) as CS+ and CS-, and a loud burst of white noise as US to evoke an affective response. These letters were then shown again within words belonging to two different categories (birds and vegetables). Two groups were tested: The reappraisal group was told that one of the categories represented safety from the white noise while the other remained dangerous. The extinction group was not told anything else and received no further bursts of white noise.

In the reappraisal group we expected to find increased SCRs in response to dangerous CS+ compared to safe CS+ and CS- trials.

In the extinction group we expected to find increased SCRs to CS+ compared to CS- trials in the early part of this phase, and no difference in the late part of this phase.

In the reappraisal group we showed an effective reduction of SCR in response to a previously conditioned letter when this letter was presented within a word belonging to a category participants knew was safe compared to a word which implied the danger of receiving a loud burst of white noise. This suggests that participants were able to reappraise this type of stimulus on the basis of cognitive evaluation of contextual information in order to determine its meaning, and that this modulated the $\mathrm{CR}$, confirming our hypothesis. One possible explanation of this result is that mechanisms similar to those engaged in classical extinction may be at the basis of this $C R$ reduction. This includes remembering the new contingencies, applying this knowledge on a trial by trial basis, and inhibiting the CR during safe trials.

In contrast, we found no significant difference between CS+ and CS- trials in the group that received the extinction conditions. We expected a difference in the early trials with the CS+ trials resulting in higher SCRs compared to CS- trials. The fact that we did not find this effect may be explained by a flaw in the design of this study, which would impact the extinction group in particular: The trials presented during the conditioning phase visually differed greatly from those presented in the extinction/reappraisal phase. In the conditioning phase we presented single letters, whereas in the extinction/reappraisal phase, words were presented for the entire duration of the trials. Further, while reappraisal group participants were informed of the danger of the aversive sound for one of the word categories when combined with the CS+, extinction participants were only asked to complete the accompanying task (to press one button if the word on the screen belonged to one of the two categories and contained the CS+, and the other button if the word belonged to one of the two categories and contained the CS-) with no further information and no further noise bursts. 
Because context is a vital factor in the maintenance of a conditioned effect (Bouton, 2004a), it is possible that the large change in context, coupled with no explicit instruction to pay attention to the combination of letter and word category, immediately abolished the conditioned response in the extinction group. In addition, we used a 100\% reinforcement schedule during conditioning, thus, even if the conditioned effect persisted in the extinction group to start with, only few trials contained enough information for this to be abolished. This factor was taken into consideration in later versions of this task.

There are several potential limitations to this task and its results. As mentioned above, the trials in the conditioning and extinction phase were perceptually quite different, even though the conditioned letters were emphasised within the words. Thus, it is possible that the trials in the extinction phase were not similar enough to maintain a conditioned response when participants were not instructed of the continued threat associated with one of the letters. In future versions of the task this will be addressed by presenting the full words for only a short period of time, keeping only the letter on screen for the remainder of each trial.

In addition, while the $100 \%$ reinforcement schedule employed in the conditioning phase may have ensured that participants were aware of the contingencies during conditioning, it may have also played a role in further speeding up the extinction process. This would make the extinction process difficult to capture in SCR which is subject to great individual differences and trial by trial variability (Cacioppo et al., 2007) and, thus, differences between conditions can only be captured with a larger number of trials. Since a $50 \%$ reinforcement schedule is an effective way to train an association between a CS+ and a US (thus, producing a CR) and slowing down the extinction process across a larger number of trials (e.g. Phelps, Delgado, Nearing, \& LeDoux, 2004), we will employ this in future task versions. Another issue was the small number of analyseable trials in this version of the task. Only 10 dangerous $\mathrm{CS}+$ trials were presented to participants, therefore only 5 of those were nonreinforced trials and thus, uncontaminated by the white noise burst. As mentioned above, SCR is highly variable between individuals so the small number of trials will have reduced our power to find an effect. We will increase the number of trials to include in the analysis in future versions and make them equal across conditions. A new version of this task is piloted below in study 2 of this paper. 


\section{Study 2: Selective Extinction through Cognitive Evaluation task}

\section{PARADIGM IMPROVEMENT AND PILOT}

\section{INTRODUCTION}

Although the results from study 1 were a promising indication that the SECE task works as intended, several issues with the design needed to be improved on to be able to draw better conclusions. This short report will go through some of the problems and explain the changes we made to the task. It will then introduce a pilot study to test a new task design.

Issue 1 - Conditioning checks not possible because CS+ reinforced $100 \%$ of the time We employed this design in the first study to make sure that participants were absolutely aware of the contingencies for the second part of the study. However, a 50\% reinforcement schedule has been shown to be as effective for learning (e.g. Phelps, Delgado, Nearing, \& LeDoux, 2004) and because of the added uncertainty on each trial they may actually be seen as more aversive than a $100 \%$ reinforcement schedule. In addition, the $100 \%$ reinforcement schedule prevented us from assessing conditioning effects because all CS+ trials were confounded by white noise. Thus, in the next part of this project, we employed a $50 \%$ reinforcement schedule both in the conditioning and the reappraisal phase.

\section{Issue 2 - Visual difference between trials in conditioning and reappraisal/extinction phase}

Trials in the conditioning phase consisted of only the letter, whereas in the reappraisal phase the word was on the screen for the duration of the trial. Although the letter was emphasised within the word trials, this visual difference may have had an important influence on the way the CS letters in the SECE/extinction phase were perceived. Especially the fact that there was no difference between CS+ and CS- trials in the extinction group suggests that this trial structure needs to be adjusted. We therefore decided to alter the trial structure and make it more similar to those employed in emotion regulation paradigms. Instead of showing the word for the duration of the trial, we decided to show the letter on its own at the start and at the end of the trial, with the word appearing briefly after the first $500 \mathrm{~ms}$. The aim of this new design is to emphasize the letter as the primary signal of danger or safety, and the word category as additional information.

\section{Issue 3 - Instructions}

Before the SECE phase, participants were told the contingencies of the task including which word category was safe and which was dangerous. Although this ensured that participants were absolutely aware of the contingencies of the task, it did not facilitate any learning and may have contributed to habituation towards the end of the task. Thus, in the next instalment of the task we only told 
participants that there was one safe and one dangerous category but not which one was which. The aim was to increase the uncertainty during the task and prolong the affective responses to the different trial types. In addition, this change enabled us to include baseline trials at the beginning of the SECE phase (before the safe - dangerous contingencies could be worked out by participants) to assess whether the conditioning effect was maintained when words were added to the trials.

\section{Issue 4 - Between groups design}

We initially chose an independent measures design because we could not counterbalance the reappraisal and extinction phases without influencing the responses differentially. In the new task design we decided to add trials which only included the letter but no reinforcement to investigate how conditioned responses change in these circumstances.

\section{Issue 5 - Sound as the US}

Although the sound was rated as aversive by all participants, because the aim was to further examine this paradigm within an MRI environment, we were unsure about its effectiveness in interaction with the MR sounds. We therefore decided to move to electric shocks as US'.

\section{METHOD}

\section{PARTICIPANTS}

5 participants ( 3 female, mean age 25.4) participated in this pilot study. They were recruited through word of mouth at the University of Reading.

\section{DESIGN}

The conditioning phase was conducted as a 2 level (CS+ vs CS-) repeated measures design, whereas the SECE phase was constructed in a $2 \times 3$ (condition (CS+ vs CS-) $x$ trial type (Safe vs Dangerous vs Letter Only)) repeated measures design. 


\section{APPARATUS}

20 trials were presented to participants in the conditioning phase -10 CS-, 5 reinforced CS+, and 5 non-reinforced CS+. Trials in the conditioning phase consisted of $4000 \mathrm{~ms}$ of the letters. The US was a mild electric shock that was adjusted individually and delivered through two $\mathrm{Ag}-\mathrm{AgCl}$ electrodes connected to an ADInstruments isolated stimulator built into a Powerlab 26T. A low constantvoltage $A C$ excitation of $22 \mathrm{mV}_{\text {rms }}$ at $75 \mathrm{~Hz}$ was passed through the electrodes, which were connected to a ML116 GSR Amp, and converted to DC before being digitized and stored.

In the reappraisal phase 20 CS-Safe, CS-Dang and CS+Safe stimuli, as well as 10 CS+Dangerous reinforced, CS+Dangerous non-reinforced, CS+Letter only, CS- Letter only were presented. Trials lasted for $5500 \mathrm{~ms}$. The first $500 \mathrm{~ms}$ only the letter was presented, then the word appeared for $1000 \mathrm{~ms}$. Finally, the word disappeared again and only the letter stayed on the screen. Throughout

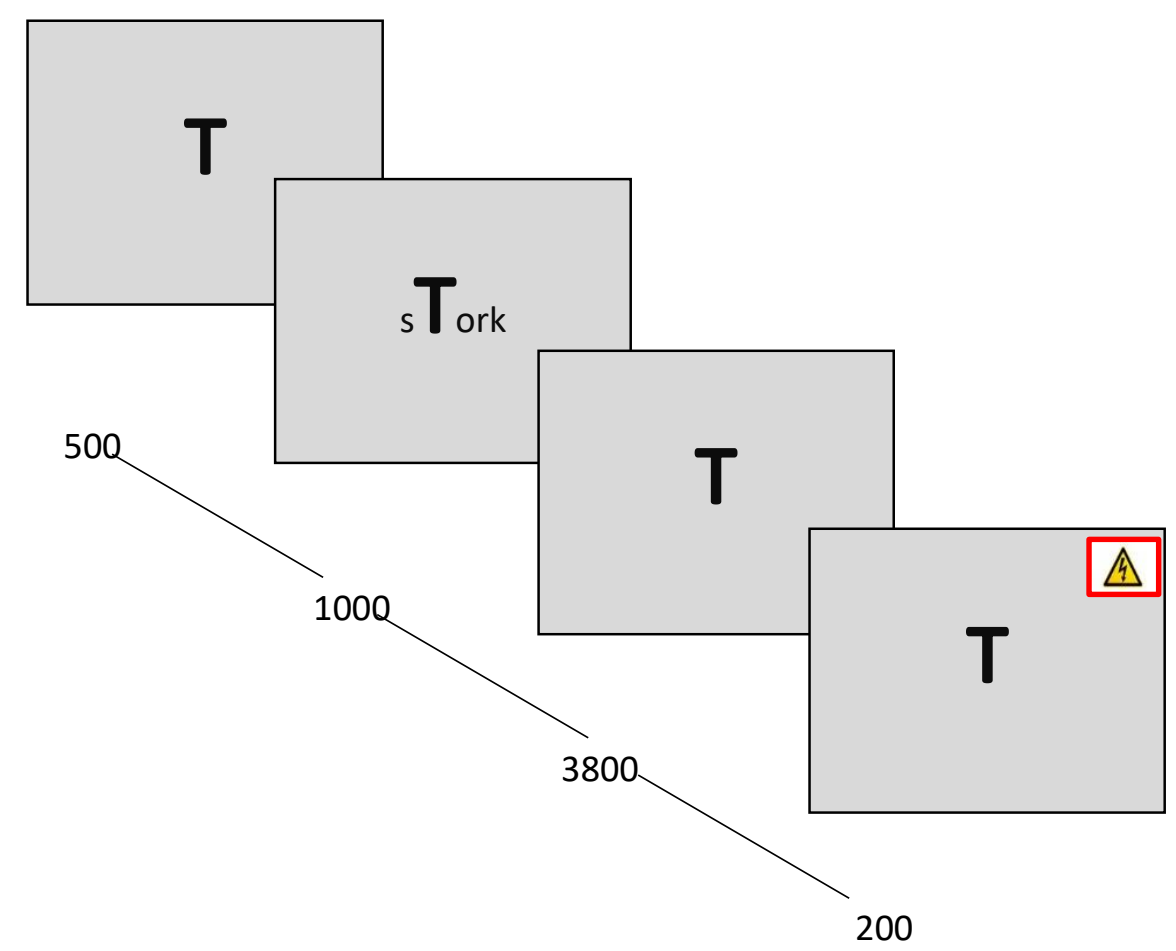

FIGURE 3. EXAMPLE OF AN SECE TRIAL.

the trial, the letter was emphasised in large and bold font (see figure 1 for an example trial). Intertrial intervals were jittered and ranged from $2000 \mathrm{~ms}$ to $6000 \mathrm{~ms}$.

Stimuli were presented using EPrime 2.0 (Psychology Software Tools, Pittsburgh, PA).

Skin conductance was recorded at $1000 \mathrm{hz}$ using a different set of $\mathrm{Ag}-\mathrm{AgCl}$ electrodes attached to the same ADInstruments Powerlab 26T via a PowerLab ML116 SCR amplifier input module.

\section{PROCEDURE}

After informed consent was obtained from each participant, the shock intensity was determined. An electric shock at a low intensity was delivered $(0.5 \mathrm{mV})$ and the intensity was increased in steps of $0.5 \mathrm{mV}$. After each shock, the participant was asked to rate the sensation on a scale of 1 ("not painful 
at all") to 10 ("extremely painful"). When they reached 8 on this scale, the intensity of the shock was reduced by 1 step and informed that this was the intensity the shock would remain at for the duration of the experiment.

Participants were then told to expect a shock to occur with one of the $\mathrm{CS}^{\prime}$ during the conditioning phase, and also that one category was safe in the reappraisal phase. They were not given any further details.

After the task, participants were debriefed and thanked for their time.

\section{SCR DATA ANALYSIS}

SCR data was analysed using a Matlab script that determined the largest deflection for each trial from a pre-trial baseline within a 7 second time window from trial onset. The data was then examined visually and outliers were removed on a trial by trial basis. These peak values were then averaged across trials for each condition for each participant.

\section{RESULTS}

Because of the limited amount of data in this pilot, no inferential statistics were carried out on these data and descriptive statistics are presented instead. We calculated standard errors across all trials, conditions and subjects to give an idea of the overall spread of the data.

The means in the conditioning phase were as expected: (Non-reinforced) CS+ trials (Mean SCR = $0.14 \mu \mathrm{S}$ ) evoked higher SCR's than CS- trials (Mean SCR $=0.11 \mu \mathrm{S}$, see figure 2 ).

Of particular interest in the reappraisal phase was the contrast between dangerous and safe CS+ trials. The pattern was as expected: dangerous CS+ trials evoked higher SCR's than safe CS+ trials $(C S+$ Dang Mean $=0.16 \mu S ; C S+S a f e$ Mean $=0.004 \mu S)$.

The negative mean of the CS+ Letter Only trial $(-0.06 \mu \mathrm{S})$ is surprising, however, because of the small number of trials and participants, this is likely to represent an artefact. No further shocks were given during these trials so this may represent an extinction effect. 


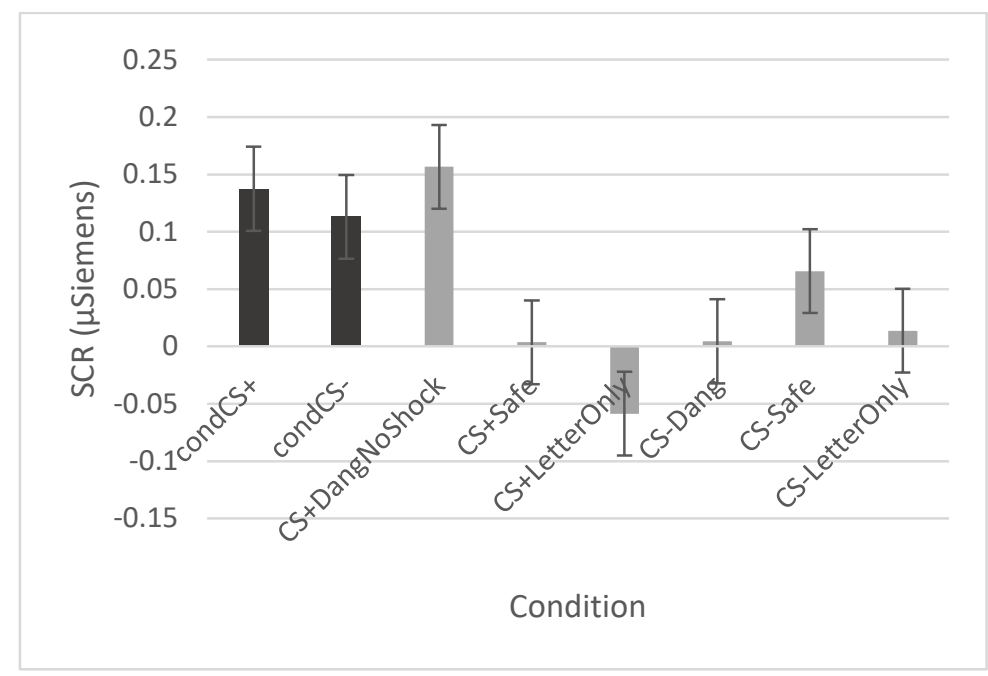

FigURE 4. MEAN SCR IN THE PILOT STUDY.

THE RESULTS POINT IN THE EXPECTED DIRECTION. SCR TO CS+ TRIALS ARE LARGER THAN THOSE TO CS- TRIALS DURING CONDITIONING. DURING THE SECE PHASE, RESPONSES TO DANGEROUS CS+ TRIALS ARE LARGER THAN THOSE TO SAFE CS+ AND ALL CS- TRIALS. ERROR BARS REPRESENT STANDARD ERRORS ACROSS ALL RESPONSES.

\section{STUDY 2 - DISCUSSION AND NEXT STEPS}

In this pilot study we found the pattern of results that we expected based on the theory of the task. Participants seemed to be able to reduce their affective response to CS+ trials when they were presented with a context based "clue" that indicated no threat of shock. This suggests that CR's were successfully produced during the conditioning phase, and that participants were able to partially extinguish them during safe trials in the SECE phase. In addition, there doesn't seem to be a difference between safe and dangerous CS- trials. Taken together, this suggests that both letter and word category cues are processed by participants. This is a good basis for further investigation including the collection of BOLD fMRI data. However, in the course of the analysis we came across more issues that needed to be resolved to improve the task.

\section{Issue 6 - Unequal numbers of comparable trials}

Although the move to a $50 \%$ reinforcement schedule in the conditioning phase meant that we were able to check whether the conditioning worked, it meant we ended up with unequal numbers of trials in the conditions which decreased the power to detect differences in our contrasts. Thus, we increased the number of CS+ trials to solve this problem. We took the same approach to the reappraisal phase, increasing the number of dangerous CS+ trials. One concern with this approach then, is the balance between CS+ and CS- and CS+Safe and Dangerous trials. Participants may process these trials differently according to the amount of times they have been exposed to them. This can be partly remedied with the order of trials. In study 1 we had the same number of trials for each condition in the experiment and they were presented in a fully randomised order. Because the likelihood of each trial occurring is equal, a fully randomised order is not problematic in that case. 
However, when the likelihood of one type of trial is more likely to occur, a pseudo-randomised design is more appropriate. There is evidence from similar types of tasks that a higher number of trials in one condition can be dealt with in that way (e.g. Delgado, Nearing, Ledoux, \& Phelps, 2008).

\section{GENERAL DISCUSSION}

Taken together, the results of this physiological study and pilot study were promising, suggesting that the aim to investigate emotion regulation on a basic level can be achieved with this task, and, thus, it is reasonable to take this a step further and investigate the neural circuits involved in this process.

On a neural level this process is thought to be completed through processing both within the amygdala itself (Pare \& Duvarci, 2012) as well as via inputs from the hippocampus (providing contextual information; Barrientos et al., 2002; Huff \& Rudy, 2004; Matus-Amat et al., 2004; Squire, 1992) and vmPFC (integrating contextual and stimulus information and feeding this back to the amygdala; Fullana et al., 2015). However, this idea will have to be explored further using imaging techniques such as fMRI to investigate the neural circuitry involved in this process. Previous studies found SCR reductions when a learned safety cue was added to a previously reinforced CS+ (Kong et al., 2014, Rescorla, 1969), however, these cues did not have to be cognitively evaluated by the participants in order to determine safety or danger. Thus, this study extended those findings by adding an extra evaluative layer and making the paradigm more similar to those used in studies of instructed emotion regulation (e.g. Buhle et al., 2014).

These results complement the findings by Delgado et al. (2008) who used instructed fear conditioning to elicit aversive responses to one of two coloured squares, and then instructed participants to reappraise these by thinking of something calming in nature. They found that participants were able to effectively reduce their affective responses through this strategy. On a neural level they found that in addition to amygdala and VMPFC, part of the dIPFC was also involved in the regulation of the $\mathrm{CR}$. The task introduced here provides increased control over the reappraisal process compared to Delgado et al. (2008), thus, future studies will investigate whether similar PFC areas mediate the reduction of the $\mathrm{CR}$ in this paradigm.

Similarly, Hefner, Verona, and Curtin, (2016) told participants to expect an electric shock after a word printed in red but not if that word belonged to one specific category. They found decreased startle and faster reaction times during safe CS+ trials. Analysis of ERP components revealed, that threat information relating to word colour (i.e. CS+) was processed before the safety signal (i.e. word category), suggesting independent analysis of the components that make up this task. In our uninstructed conditioning task we found a similar effect -SCR's in response to both safe and dangerous CS+ trials were increased compared to CS- trials, and dangerous CS+ trials produced larger 
SCR's than safe CS+ trials. This also suggests that the information related to the letters (CS) had an influence on SCR independently from the information related to the word (safety cue).

\section{CONCLUSION}

This study used a novel task to assess the impact of simple cognitive evaluations on autonomic responses to letter stimuli that had previously been conditioned to an aversive white noise. The results suggest that participants were able to reappraise the CS+ and CR's were significantly reduced when the CS+ was presented with additional information they knew to predict safety from the white noise burst. As a next step, this paradigm will be improved by addressing the limitations mentioned above including changing the reinforcement schedule to $50 \%$ during conditioning, redesigning the trials in the extinction phase to be perceptually more similar to the conditioning trials, and increasing the number of trials over all. In addition, we will go one step further in investigating whether extinction mechanisms are applied during this task and collect BOLD fMRI in addition to SCR.

\section{REFERENCES}

Barrientos, R. M., Higgins, E. A., Sprunger, D. B., Watkins, L. R., Rudy, J. W., \& Maier, S. F. (2002). Memory for context is impaired by a post context exposure injection of interleukin-1 beta into dorsal hippocampus. Behavioural Brain Research, 134(1), 291-298.

https://doi.org/10.1016/S0166-4328(02)00043-8

Battig, W. F., \& Montague, W. E. (1969). Category norms of verbal items in 56 categories A replication and extension of the Connecticut category norms. Journal of Experimental Psychology Monograph, 80(3), 1-46.

Bouton, M. E. (2004). Context and Behavioral Processes in Extinction. Learning \& Memory, 11(5), 485-494. https://doi.org/10.1101/Im.78804

Bouton, Mark E. (2002). Context, ambiguity, and unlearning: sources of relapse after behavioral extinction. Biological Psychiatry, 52(10), 976-986. https://doi.org/10.1016/S00063223(02)01546-9

Buhle, J. T., Silvers, J. A., Wager, T. D., Lopez, R., Onyemekwu, C., Kober, H., ... Ochsner, K. N. (2014). Cognitive reappraisal of emotion: a meta-analysis of human neuroimaging studies. Cerebral Cortex (New York, N.Y. : 1991), 24(11), 2981-2990. https://doi.org/10.1093/cercor/bht154

Cacioppo, J. T., Tassinary, L. G., \& Berntson, G. G. (2007). Handbook of Psychophysiology (3rd ed.). New Yor, NY: Cambridge University Press.

Davey, G. C. . (1992). Classical conditioning and the acquisition of human fears and phobias: A review 
and synthesis of the literature. Advances in Behaviour Research and Therapy, 14(1), 29-66. https://doi.org/10.1016/0146-6402(92)90010-L

Delamater, A. R. (2010). Experimental extinction in Pavlovian conditioning: Behavioural and neuroscience perspectives. Quarterly Journal of Experimental Psychology Section B. Retrieved from http://www.tandfonline.com/doi/abs/10.1080/02724990344000097\#.VcSuzvlViko

Delgado, M. R., Nearing, K. I., Ledoux, J. E., \& Phelps, E. A. (2008). Neural circuitry underlying the regulation of conditioned fear and its relation to extinction. Neuron, 59(5), 829-838. https://doi.org/10.1016/j.neuron.2008.06.029

Fullana, M. A., Harrison, B. J., Soriano-Mas, C., Vervliet, B., Cardoner, N., Àvila-Parcet, A., \& Radua, J. (2015). Neural signatures of human fear conditioning: an updated and extended meta-analysis of fMRI studies. Molecular Psychiatry. https://doi.org/10.1038/mp.2015.88

Grillon, C., \& Ameli, R. (2001). Conditioned inhibition of fear-potentiated startle and skin conductance in humans. Psychophysiology, 38(05), 807-815. Retrieved from http://journals.cambridge.org/abstract_S0048577201000294

Gross, J. J., \& John, O. P. (2003). Individual differences in two emotion regulation processes: implications for affect, relationships, and well-being. Journal of Personality and Social Psychology, 85(2), 348-362. Retrieved from http://doi.apa.org/getdoi.cfm?doi=10.1037/00223514.85.2.348

Hefner, K. R., Verona, E., \& Curtin, J. J. (2016). Emotion regulation during threat: Parsing the time course and consequences of safety signal processing. Psychophysiology, 53(8), 1193-1202. https://doi.org/10.1111/psyp.12660

Huff, N. C., \& Rudy, J. W. (2004). The Amygdala Modulates Hippocampus-Dependent Context Memory Formation and Stores Cue-Shock Associations. Behavioral Neuroscience, 118(1), 53-62. https://doi.org/10.1037/0735-7044.118.1.53

Kilgarriff, A. (1997). Putting frequencies in the dictionary. International Journal of Lexicography, 10(2), 135-155. https://doi.org/10.1093/ijl/10.2.135

Kong, E., Monje, F. J., Hirsch, J., \& Pollak, D. D. (2014). Learning not to fear: neural correlates of learned safety. Neuropsychopharmacology : Official Publication of the American College of Neuropsychopharmacology, 39(3), 515-527. https://doi.org/10.1038/npp.2013.191

Matus-Amat, P., Higgins, E. A., Barrientos, R. M., \& Rudy, J. W. (2004). The Role of the Dorsal Hippocampus in the Acquisition and Retrieval of Context Memory Representations. Journal of 
Neuroscience, 24(10).

McRae, K., Ciesielski, B., \& Gross, J. J. (2012). Unpacking cognitive reappraisal: Goals, tactics, and outcomes. Emotion, 12(2), 250-255. https://doi.org/10.1037/a0026351

Mechias, M.-L., Etkin, A., \& Kalisch, R. (2009). A meta-analysis of instructed fear studies: Implications for conscious appraisal of threat. Neurolmage, 49, 1760-1768.

https://doi.org/10.1016/j.neuroimage.2009.09.040

Meyer, T. J., Miller, M. L., Metzger, R. L., \& Borkovec, T. D. (1990). Development and validation of the penn state worry questionnaire. Behaviour Research and Therapy, 28(6), 487-495.

https://doi.org/10.1016/0005-7967(90)90135-6

Pare, D., \& Duvarci, S. (2012). Amygdala microcircuits mediating fear expression and extinction. Current Opinion in Neurobiology. Retrieved from http://www.sciencedirect.com/science/article/pii/S0959438812000372

Pavlov, I. (1927). Conditioned reflexes. New York, NY, US: Dover.

Phelps, E. A., Delgado, M. R., Nearing, K. I., \& LeDoux, J. E. (2004). Extinction learning in humans: role of the amygdala and vmPFC. Neuron, 43(6), 897-905. https://doi.org/10.1016/j.neuron.2004.08.042

Rescorla, R. A. (1969). Pavlovian conditioned inhibition. Psychological Bulletin, 72(2), 77-94.

Squire, L. R., \& R., L. (1992). Memory and the hippocampus: A synthesis from findings with rats, monkeys, and humans. Psychological Review, 99(2), 195-231. https://doi.org/10.1037/0033295X.99.2.195

Watson, D., Clark, L. A., \& Tellegen, A. (1988). Development and validation of brief measures of positive and negative affect: the PANAS scales. Journal of Personality and Social Psychology, 54(6), 1063-1070. Retrieved from http://www.ncbi.nlm.nih.gov/pubmed/3397865 